Courrier du Centre international Blaise Pascal

19 | 1997

Varia

\title{
Études pascaliennes au Japon depuis 1989
}

\section{Hiroto Nishikawa}

\section{OpenEdition}

Journals

Édition électronique

URL : http://journals.openedition.org/ccibp/565

DOI : $10.4000 /$ ccibp.565

ISSN : 2493-7460

\section{Éditeur}

Centre international Blaise Pascal

\section{Édition imprimée}

Date de publication : 5 avril 1997

Pagination : 33-36

ISSN : 0249-6674

\section{Référence électronique}

Hiroto Nishikawa, «Études pascaliennes au Japon depuis 1989 », Courrier du Centre international Blaise Pascal [En ligne], 19 | 1997, mis en ligne le 06 janvier 2016, consulté le 14 septembre 2020. URL http://journals.openedition.org/ccibp/565

Ce document a été généré automatiquement le 14 septembre 2020.

Centre international Blaise Pascal 


\title{
Études pascaliennes au Japon depuis 1989
}

\author{
Hiroto Nishikawa
}

1 Sur les études pascaliennes au Japon depuis 1989, M. Maeda a fait paraître un rapport intitulé Pascal au Japon dans Chronique de Port-Royal, $n^{\circ} 22-23$, en 1974 (1 $1^{\text {re }}$ époque). Nous l'avons suivi et avons publié un compte rendu, Études pascaliennes depuis 1974, dans Pascal Port-Royal Orient Occident, Actes du colloque de l'université de Tokyo, 27-29 septembre 1988 ( $2^{\mathrm{e}}$ époque). Pour la deuxième époque, nous avons fait remarquer deux tendances : la traduction et l'étude originale.

De fait, depuis 1989, nous avons des résultats originaux comme on s'y attendait. Quelques-uns ont essayé d'entendre des voix différentes dans les Pensées comme apologie. M. Hisashi Suematsu a publié Voix dans le discours apologétique des Pensées (1990, en japonais, Éditions de l'université de Kyushu). Dans ce livre, ayant expliqué beaucoup des fragments des Pensées, il a développé le même thème qu'il avait exposé au Colloque de l'université de Tokyo. En essayant de lire l'apologie pascalienne comme œuvre littéraire et œuvre d'art verbale, il fait observer les voix diverses des personnages fictifs que l'apologiste met en scène dans les Pensées. En un mot, M. Suematsu entend les voix différentes des interlocuteurs fictifs dans les Pensées.

Spécialiste de la philosophie, après avoir fait des études sur Schopenhauer et Nietzsche, M. Masayuki Kodama, a publié La vision pascalienne de l'homme - ni ange ni bête (1992, en japonais, Éditions Kôrosha) qui se compose en trois parties : I. « La science, l'art et l'histoire dans la pensée de Pascal fondée sur les trois concupiscences », II. «L'étude de l'homme par Pascal », III. «Le pari et le christianisme chez Pascal ». C'est pour vérifier si la phrase de Nietzsche "le christianisme a ruiné la plus forte et la plus noble âme (Pascal) » (La volonté de puissance, 252) est juste ou non qu'il se plonge dans les études sur Pascal. Il se demande si Nietzsche a précisément compris Pascal. D'abord, il commence à éclaircir la pensée pascalienne sur la science, sur l'art, etc., fondée sur les trois concupiscences, pour chercher quelle est la vision de l'homme de Pascal.

Ensuite, suivant Goldmann, il trouve dans les Pensées la vision tragique et paradoxale de l'homme qui, en courant après la valeur absolue, ne peut jamais la trouver dans sa vie 
terrestre. C'est le point de vue de l'unité dialectique pascalienne qui, reniant le dogmatisme et le pyrrhonisme, les dépasse tous les deux. M. Kodama y trouve l'essence de Pascal croyant, qui réside dans son cœur même.

Dans la troisième partie, il traite de la question du pari. Par l'analyse de la pensée de Pascal qui a introduit dans le domaine de la foi « la règle des partis ", ce spécialiste de la philosophie s'approche de la véritable intention de Pascal dans sa critique de saint Augustin (L.577, S. 480). Ici aussi, d'après l'avis de Goldmann, M. Kodama fait remarquer que Pascal veut représenter "la structure paradoxale de la nature et de Dieu » contre les croyants qui sont convaincus que « la religion est sûre ».

M. Tetsuya Shiokawa a fait paraître Arc-en-ciel et sacrement (1993, en japonais, Éditions Iwanami) qui se compose en trois chapitres : I. «Traduction des voix », II. «Théorie de l'arc-en-ciel, III. «Structures de la foi ». D'abord, en général, observe-t-il, les œuvres littéraires ont une identité ou une unité par le titre de l'œuvre donné par l'auteur, mais dans le cas des Pensées, le titre, ainsi que le texte exact de l'œuvre, ne sont pas établis par Pascal. L'existence de ses manuscrits ne donne pas accès à l'édition définitive, mais au mieux, aux meilleures éditions possibles. En somme, ce sont des textes privés d'identité parfaite, impossibles à déterminer, à identifier. A cet égard, les lecteurs des Pensées se demandent si la voix qu'ils y entendent sont toujours vraiment celle de Pascal lui-même ou bien celles de plusieurs interlocuteurs. M. Shiokawa considère l'attitude de l'auteur des Pensées, qui a connu, lors de la nuit de feu, la présence de Dieu, comme celle d'un prophète qui dit le message de Dieu. Et il analyse ainsi : les chrétiens, tout en croyant que le pain se transsubstantie en corps du Christ par les paroles consacrées et que la Présence réelle s'accomplit pour eux, ne peuvent voir ni comprendre cela par la raison; de même les lecteurs des Pensées éprouvent que ces textes privés de l'identité parfaite et de l'unité sont des fragments d'une expérience de la Présence et que chaque fragment contient à la fois cette expérience tout entière.

7 Ensuite, M. Shiokawa considère en détail les questions des signes et des figures dans $L a$ logique de Port-Royal (I, 4). Il fait remarquer que dans ces questions on voit les conséquences des controverses sur la Présence réelle entre Arnauld et les protestants. À propos de l'arc-en-ciel comme signe (Genèse, IX, 23), Arnauld et Nicole connaissent bien l'explication cartésienne sur la nature de l'arc-en-ciel, et la contradiction n'existe pas pour eux entre l'explication scientifique sur la nature de ce phénomène et la leçon biblique sur la signification de l'arc-en-ciel. Car « que les couleurs soient réelles ou non réelles, ne suffit-il pas que nos yeux soient frappés de la vue de cette figure dans les nues, pour se souvenir de la promesse de Dieu?» (La perpétuité de la foi de l'Église catholique, 1781, t. III, liv. III, ch. 1, p. 142). En somme M. Shiokawa montre, dans la discussion sur les signes, les exemples qui semblent ruiner la valeur des signes comme signes et leur valeur sémantique. C'est qu'à peine les figures dans la nature ou dans l'histoire - originaux révélés par la copie - sont-elles identifiées, qu'elles deviennent invisibles. Et il conclut que c'est à rendre la réalité aux figures par la supposition nécessaire des originaux invisibles que vise l'abnégation par la foi de Pascal.

Dans le dernier chapitre, après avoir poursuivi la structure de foi dans La logique (IV) et à propos de la signature du formulaire etc., il indique que Pascal a nommé « notre état de foi » (Seizième Provinciale) l'état d'âme des chrétiens qui sont en face de la figure contenant à la fois la présence et l'absence. Il conclut que cet « état de foi » est le plus convenable à tous les hommes qui ne peuvent s'empêcher de chercher la Présence impossible à atteindre, et que la foi n'est que l'expression de la condition de l'homme. 
9 En 1992, a paru un livre important, Pascal, de l'intuition à l'affirmation: Processus de l'achèvement de ses traités de physique (en japonais, thèse de doctorat de l'université d'Osaka, Éditions de l'université de Nagoya) de $\mathrm{M}^{\mathrm{me}}$ Kimiyo Koyanagi. Cette œuvre se compose en cinq parties : I. « Expériences des Italiens », II. « Pascal à Rouen », III. « Les savants parisiens et la colonne d'air", IV. "Rédaction du grand traité », V. «Achèvement et rejet des deux traités ». D’abord, elle lit attentivement le fragment du Traité $d u$ vide de Pascal, et elle aperçoit des incohérences entre les rapports des expériences de Pascal et le tableau récapitulatif qu'il donne de ses résultats. Et puis elle compare les rapports d'expériences des Italiens et ceux de Pascal à Rouen et à Paris etc. Et en supposant que Pascal non seulement a fait des expériences réelles mais s'est appuyé aussi sur des expériences fictives par le raisonnement, elle refait par elle-même les expériences pascaliennes sur le vide et sur les liqueurs. Les résultats confirment son hypothèse que Pascal s'est appuyé sur des expériences fictives par le raisonnement. Elle énumère les résultats de beaucoup d'expériences du temps de Pascal et de réexpériences faites par elle-même et par ses collègues.

Ensuite, elle examine à quelle époque Pascal a lu le rapport de Torricelli et estime que c'est en 1651 qu'il a achevé ses Traités de l'équilibre des liqueurs et de la pesanteur de la masse de l'air. Sa conclusion, c'est que l'originalité éminente de Pascal réside dans le fait qu'il a deviné d'un seul trait l'essence des choses par son intuition géniale et qu' « il a mis en ordre quelques principes trouvés en désordre ». Grâce au résumé en anglais à la fin de l'ouvrage, les lecteurs occidentaux peuvent connaître les points essentiels de ce livre.

11 L'été 1990 a paru un numéro spécial sur Pascal de la revue Équinoxe (en français, RinsenBooks), conçu par M. Shiokawa. On y trouve dix articles. Dans le premier, « Pascal et la spiritualité des Chartreux ", M. Mesnard montre, par des rapprochements avec Pharetra (Discours en forme de Lettre de Notre Seigneur Jésus-Christ) de Lanspergius, prieur de la chartreuse de Juliers, les rapports étroits entre cette œuvre et quelques fragments des Pensées de Pascal : L. 919 - S. 751, L. 929 - S. 756, L. 931 - S. 759, L. 360, 368 374 - S. 329, 401 406, L. 113, 200 - S. 145, 231, etc. M. Mesnard fait remarquer aussi les mêmes influences dans les lettres $M^{\text {lle }}$ de Roannez. Et il conclut que Pascal « emprunte des cadres aux Chartreux, mais les remplit d'un contenu qui lui est propre ».

Dans Les premières Provinciales et le dialogue d'idées au XVII ${ }^{e}$ siècle, M. Sellier traite de l'apparition des dialogues d'idées au XVII ${ }^{\mathrm{e}}$ siècle, et montre l'influence de Platonconcis ou abrégé. "Enfin avait paru le Platon qu'il convenait à «impatience » française »! Surtout, dit-il, les aspects divers (ironie, interrogations proposées par un faux ignorant, etc.) des premières Provinciales s'inscrivent «à l'intérieur de ce mouvement qui tentait de réconcilier philosophie ou théologie et l'élaboration littéraire. "

13 M. Shiokawa, dans Imagination, fantaisie et opinion: pourquoi Pascal prend-il pour thème l'«imagination » dans le fragment 44-78 des Pensées ?, se demande pourquoi ce fragment est inséré par Pascal dans la liasse intitulée "Vanité » et il s'interroge sur l'usage du mot Imagination, dans le contexte intellectuel du XVII ${ }^{\mathrm{e}}$ siècle. Il consulte non seulement les dictionnaires et les textes des auteurs de cette époque, mais aussi les textes d'Aristote, d'Épictète, de Montaigne etc. et conclut que ce fragment « illustre, lui aussi, la mise en pratique de ce projet de destruction symétrique du dogmatisme stoïcien et du scepticisme épicurien ». 

œuvres de Pascal sur le vide et l'hydrostatique»; Christian Meurillon, "Les combinaisons pascaliennes ou les avatars de la pensée ternaire »; Anthony McKenna, «Coutume/Nature : la fortune d'une pensée de Pascal»; Gérard Ferreyrolles, "Pascal critique de la coutume »; Tomohiro Ishikawa, «La théorie des trois états de l'homme chez Pascal »; Vincent Carraud, «L'égologie cartésienne subvertie : le fragment 688 des Pensées »; Hisashi Suematsu, «Discours de De la sagesse ». Cette revue peut se procurer aux éditions Slatkine (Genève). dans les revues scientifiques. D'abord, M. Takaharu Hasekura, succédant à M. Maeda, continue de faire les commentaires détaillés des Pensées de Pascal (de L. 203 - S. 235 à L. 209 - S. 241, 242) depuis 1988 dans Études de langue et littérature française de l'université de Tokyo, $n^{\text {os }} 35-43$. On attend leur publication en forme de livre le plus tôt possible.

Dans la revue Shiso (Pensée), ont été publiées, en 1989, la traduction japonaise de la Théologie de la grâce et sciences de l'homme de J. Mesnard (conférence prononcée à l'université Sophia de Tokyo 1988), et l'année suivante, celle de la conférence Culture féminine et littérature classique de M. Sellier (prononcée également à l'université Sophia de Tokyo). littéraires en France, M. Shiokawa a publié un article intitulé a Rhétorique et histoire littéraire - à propos de la Onzième Provinciale ». Dans cette Onzième Provinciale, Pascal critique une ode intitulée Éloge de la pudeur du jésuite Pierre Le Moyne. Sa critique, dit M. Shiokawa, révèle la piété de Port-Royal qui contraste avec l'impiété ou la frivolité de ce père. Cette campagne est profondément liée avec la rivalité bien connue entre les courants baroque et classique de la rhétorique, mais aussi entre la rhétorique des jésuites et celle des gallicans, comme l'observe M. Fumaroli. Et M. Shiokawa fait remarquer que la rhétorique au sens profond ne consiste donc pas seulement à exprimer une réalité identique de plusieurs manières différentes, mais que le choix d'un mode d'expression correspond à un choix idéologique dans les divers domaines de la morale, de la politique, de la religion, etc.

18 En 1990, l'université Kwansei Gakuin a publié, dans la revue Sengari, les conférences qui se sont tenues au Centre des séminaires en 1988, comme suit: Jean Mesnard, Les Pensées de Pascal, trésor de l'occident et Science et religion chez Pascal (en français avec traduction japonaise); Philippe Sellier, Sur les fleuves de Babylone : la fluidité du monde et la recherche de la permanence dans les Pensées (en français avec traduction japonaise); Kokiti Hara, Pascal dans l'histoire des mathématiques (en français); Thérèse Goyet, Dans le sillage de Pascal, l'initiation japonaise (en français).

19 Dans notre article intitulé Sur Le Mémorial de Pascal - son expérience mystique et l'expression de cette expérience (in Sophia de l'université Sophia, 1991, en japonais), après avoir fait des recherches sur les expériences mystiques de St Jean de la Croix, de Ste Thérèse d'Avila et de Pascal, nous formulons une hypothèse sur la raison pour laquelle Pascal n'a confié à personne son expérience de la nuit de feu et sur la manière dont il l'a exprimée néanmoins.

Voici les titres des autres articles dans les bulletins ou dans les revues: 
21 Kimiyo Koyanagi, «Soixante et onze docteurs : à propos d'une phrase de la Première Provinciale ", (in Bulletin Annuel de la Société de Langue et Littérature Française du Chûbu, $\mathrm{n}^{\circ}$ 14, 1990, en japonais) ; Hajime Morikawa, « Annuae Litterae Prouinciae Ad annum Christi 1656 et Les Lettres Provinciales de Pascal » (in Gallia, XXXI, 1991, partie d'une thèse de doctorat de l'université d'Osaka, en japonais); Haruo Nagase, «Les Pensées et l'opposition des termes binaires»(Id.) ; Yuka Mochizuki, "Sur les deux feuilles de manuscrits du «Pari » de Pascal » (in Études de langue et littérature française, n 60, 1992, en français); Id., Autorité langue. "À propos de l'interprétation d'Arnauld et de Pascal sur les décisions du Concile de Trente relatives à la possibilité d'accomplir les commandements » (in Études et la littérature française de l'université de Kyoto, 1992, en japonais) ; Koji Kawamata, «Les nu-pieds et Pascal » (in Bulletin d'études françaises de l'université Dokkyo, n²3, 1992, en français); Id., «Les soulèvements populaires et Pascal », (in Shiso, en japonais) ; Yoshikatsu Yoshida, « Pascal et le logos. Un essai sur le rejet de la "démonstration métaphysique " (in Mélanges intitulés Âme de la civilisation française. Sa langue et sa littérature, 1993, en japonais) ; Hiroto Nishikawa, "Sur les sources bibliques de L'Entretien avec $M$. de Sacy" (in Revue d'études françaises de l'université Sophia, n²7, 1993, en japonais) ; Hajime Morikawa, « Les Lettres Provinciales et Annuae Litterae Prouinciae Franciae Ad annum Christi 1656 (in Kwanseigakuin University Annual Studies, vol. XLIII, 1994, en français); Hisashi Suematsu, «Discours sur les passions de l'amour. Un essai sur la sémiotique du sexe (I)» (in Études de la littérature de l'université de Kyushu, 1996, en japonais).

Dans les trois articles cités ci-dessus, M. Kawamata, après avoir parcouru les documents anciens et modernes sur les soulèvements au XVII ${ }^{\mathrm{e}}$ siècle, montre que Pascal révèle, dans divers fragments des Pensées (L. 44 - S. 78, L.60 - S. 94, L. 66 - S. 100, L. 80 - S. 115, L. 87 - S. 121, L 434 - S. 686, L. 435 - S. 687, L. 797 - S. 650, etc.), les impressions profondes qu'il a ressenties devant les soulèvements populaires.

Il faudrait mentionner spécialement le commencement de la publication de la traduction des CEuvres Complètes éditées par M. Jean Mesnard. Le tome premier a paru le 11 novembre 1993, sixième anniversaire de la mort de $M$. Maeda, et le deuxième le 4 juin 1994. Ces travaux sont poursuivis par une équipe de traducteurs sous la direction de MM. Akagi, Hasekura, Hirota et Shiokawa. Quand cette traduction sera achevée, elle fera époque dans le domaine des études pascaliennes au Japon. Pour cela aussi, on attend avec impatience l'achèvement de cette édition nouvelle de M. Mesnard.

L'année 1995 a été une année de bonheur et de grande joie pour les pascalisants japonais. Car M. Mesnard (en juin) et M. Sellier (en septembre et octobre) sont venus au Japon. En tant que missionnaire de l'École normale supérieure, M. Mesnard a prononcé des conférences sur «La fondation de l'École normale supérieure » (à l'université de Tokyo, in Revue de langue et littérature française, de l'université de Tokyo, n 14, 1996), sur «Les éditions des Provinciales » (à la Société d'Études pascaliennes au Japon), et sur "Science et foi chez Pascal» (à l'université Sophia, traduction japonaise in Sophia, $\left.n^{\circ} 176,1995\right)$. M. Sellier, invité par l'université de Tokyo, a donné des conférences et des séminaires dans plusieurs universités, parmi lesquels : Saint Augustin dans la culture du XVII ${ }^{\mathrm{e}}$ siècle français " (à l'université de Tokyo et à l'université de Kyushu, in Revue de langue et littérature française de l'université de Tokyo, $\left.\mathrm{n}^{\circ} 14,1996\right)$; "Théologie et imaginaire chez Pascal» (à la Société d'Études pascaliennes); "Qu'est-ce qu'être classique ? » et «L'Ouverture du projet d'apologie (fr. 681)» (à l'université de Tokyo) ; «L'ordre du cœur chez Pascal » (à l'université Sophia, traduction japonaise in Sophia [à 
paraître]); «L'ouverture du projet d'Apologie » et «Pascal et saint Augustin: des Confessions aux Pensées » (à l'université Kwansei Gakuin).

En 1994, quatre étudiants japonais ont obtenu leur D.E.A. à l'université de Paris-IV en faisant des recherches sur Pascal: $\mathrm{M}^{\text {lle }}$ Juri Higaki, $\mathrm{M}^{\text {me }}$ Yuka Mochizuki, MM. Hirotsugu Yamajo et Yoshikastu Yoshida. Nous attendons beaucoup de ces jeunes chercheurs.

INDEX

Mots-clés : Pascal, études

Keywords: Pascal, studies

Index géographique : Japon 